\title{
Improvement of an Arm Exoskeleton by Data Fusion with an Inertial Measurement Unit
}

\author{
Jie Chen, Canjun Yang \\ The State Key Laboratory of Fluid Power Transmission \\ and Control \\ Zhejiang University \\ Hangzhou, China \\ e-mail: ycj@zju.edu.cn
}

\author{
Jens Hofschulte \\ ABB Corporate Research China \\ Shanghai, China \\ e-mail: jens.hofschulte@cn.abb.com
}

\begin{abstract}
This paper aimed to improve the performance of a 7-DOFs arm exoskeleton in position measurement, so that it can be used for applications that require high-accuracy tracking ability, such as robot navigation. A data fusion method with an inertial measurement unit(IMU) using Kalman Filter is developed. With proper setup of the system the result of experiments show a significant improvement in position accuracy of the exoskeleton.
\end{abstract} filter

Keywords-data fusion; exoskeleton; inertial sensor; Kalman

\section{INTRODUCTION}

Arm exoskeleton is an external mechanical device worn by human arm. It is widely employed in tele-operation, rehabilitation and strength enhancement [1], [2], [3], [4]. The control accuracy of the exoskeleton is very important in certain applications such as robot tele-operation. However, due to the manufacturing error and accumulate error the series structure, the end position and orientation detected by the exoskeleton can't reach the high demand of those applications. Thus, we proposed a data fusion method using an IMU to improve the accuracy of the exoskeleton.

Inertial measurement units (IMUs) are electronic devices that measures velocity, orientation, and gravitational forces, using a combination of accelerometers and gyroscopes, sometimes also magnetometers. With the advantages of low cost, light weight, and fast response, they are widely used for navigation of vehicle/aircraft and human motion detection [5], [6], [7]. IMU has good short-term precision and high sampling rates, but it suffers from serious errors in long-term position and orientation estimates due to the drift and the algorithm of integration[8]. It has already been used to improve the performance of a GPS-based navigation system in terms of dynamic behavior, synchronization, and reliability [9], [10], [11].

Multi-sensor data fusion become increasingly popular in industry and scientific research. Besides the applications of GPS-based navigation system. There are also several multisensor systems that combine optical tracking system (OTS) and IMU developed for surgical navigation, user tracking in augmented reality, and human motion detection [12], [13], [14]. Kalman filter is a common fusion algorithm for multi- sensor data fusion, which is implemented in the proposed method for data fusion of the exoskeleton and IMU.

\section{SYSTEM SETUP}

A. Exoskeleton system

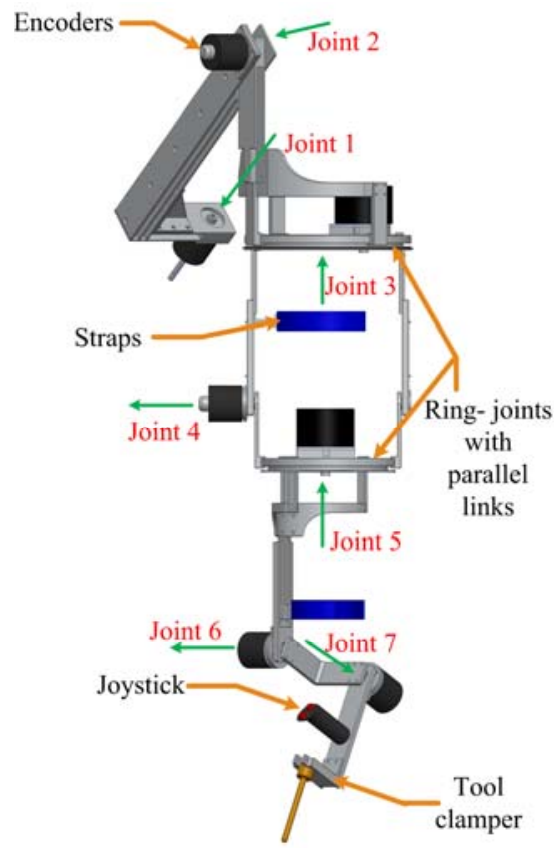

Figure 1. Structrue of the exoskeleton.

The proposed exoskeleton is designed especially for robot tele-operation. There are 7 joints from shoulder to wrist, each joint is equipped with an optical encoder which has an resolution of 12 bits. In order to simplify the mechanism, there is no driven joint which also reduce the weight and cost of the exoskeleton. The links are designed to be adjustable for different operators. In the end of the exoskeleton, there are a joystick with buttons for the control of the robot and a tool clamper for different tools. With all this optimized mechanism, the total weight of the exoskeleton is reduced to $2 \mathrm{~kg}$. 
With forward kinematics of the exoskeleton, the position and orientation of the exoskeleton's end can be calculated using the joint angles provided by the encoders

\section{B. IMU system}

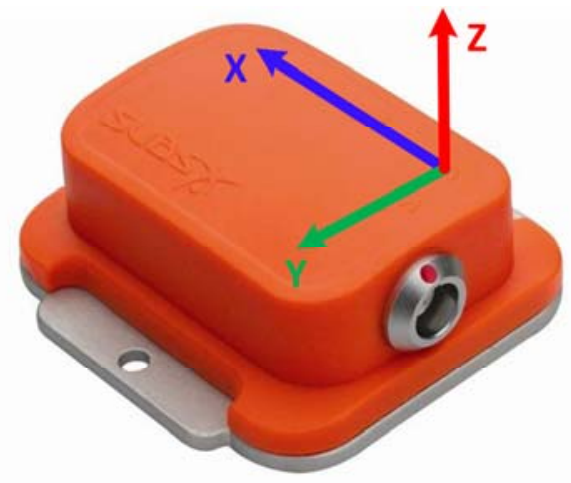

Figure 2. Xsens MTi inertial measurement unit.

An MTi (Xsens Technologies B.V., Enschede, The Netherlands) inertial measurement unit is used in our system, as shown in Fig. 2. It consists of a 3D MEMS acceleration sensor, a 3D MEMS gyroscope, and a 3D earth-magnetic field sensor. The IMU can provide the calibrated accelerations in 3 axes with gravity and the rates of turn in its body frame $(C F)_{i m u}$. With a combination of earth-magnetic field sensor, the IMU is able to provide the orientation of the IMU in the world's coordinate frame with an accuracy of 0.1 degree. Unfortunately, the pose estimated by IMU cannot be trusted in our case as the electric signals disturbs the magnetic field sensor. Thus, only the accelerations and the rates of turn are used in our research:

$$
{ }_{(\text {imu })} \varepsilon=\left[a_{x}, a_{y}, a_{z}, \omega_{x}, \omega_{y}, \omega_{z}\right]
$$

The IMU's maximum sample rate is $512 \mathrm{~Hz}$.

\section{DATA FUSION}

\section{A. State Models}

To track the tool at the EE of the robot, both position and orientation have to be estimated by the Kalman Filter. The data acquired from the introduced measurement sensors are given in different coordinate frames, viz. coordinate frame of the exoskeleton $(C F)_{e x o}$ and coordinate frame of the IMU $(C F)_{i m u}$ The former is treated as the world's coordinate frame $(C F)_{\text {exo }}$.

The acceleration data provided by IMU contains the gravitational acceleration $g$. Before using it for estimation, a compensation of gravitation terms $g$ should be achieved:

$$
\ddot{x}_{p}(t)=R_{\text {imu }}^{\text {exo }}(t) \ddot{x}_{i m u}(t)-g
$$

On the other hand, the turn rate provided by IMU also needs to be converted into the coordinate frame of OTS:

$$
\dot{x}_{o}(t)=R_{i m u}^{e x o}(t) \dot{x}_{i m u}(t)
$$

As a result, the position estimation not only takes the object's acceleration into account, but also the orientation matrix $R_{i m u}^{o t s}(t)$ at time t. Thus, a separate determination of the position and the orientation state space model is required. The classical differential equation for translation with acceleration and rotation with constant turn rate is:

$$
\begin{array}{r}
x_{p}=x_{p}^{i n i}+\dot{x}_{p} t+\ddot{x}_{p} t^{2} / 2 \\
x_{o}=x_{o}^{i n i}+\dot{x}_{o} t
\end{array}
$$

Thus, the state space vectors separated with position and orientation of the model are created as follows:

$$
\begin{aligned}
& {\left[\begin{array}{l}
x_{p}(t) \\
\dot{x}_{p}(t) \\
\ddot{x}_{p}(t)
\end{array}\right]} \\
& {\left[\begin{array}{l}
x_{o}(t) \\
\dot{x}_{o}(t)
\end{array}\right]}
\end{aligned}
$$

According to the classical differential equation for translation with acceleration and rotation with constant turn rate, the discretized transition equations of state space models concerning position as well as orientation result to exemplarily for one dimension:

$$
\begin{gathered}
{\left[\begin{array}{c}
x_{p}(k) \\
\dot{x}_{p}(k) \\
\ddot{x}_{p}(k)
\end{array}\right]=\left[\begin{array}{ccc}
1 & \Delta T & \Delta T^{2} / 2 \\
0 & 1 & \Delta T \\
0 & 0 & 1
\end{array}\right]\left[\begin{array}{c}
x_{p}(k-1) \\
\dot{x}_{p}(k-1) \\
\ddot{x}_{p}(k-1)
\end{array}\right]} \\
{\left[\begin{array}{c}
x_{o}(k) \\
\dot{x}_{o}(k)
\end{array}\right]=\left[\begin{array}{cc}
1 & \Delta T \\
0 & 1
\end{array}\right]\left[\begin{array}{c}
x_{o}(k-1) \\
\dot{x}_{o}(k-1)
\end{array}\right]}
\end{gathered}
$$

Where $\Delta T$ is the sample time of the discretization.

\section{B. Measurement Models}

According to the data provided by the IMU and exoskeleton, the equations for the measurement models of position and orientation are:

$$
\left[\begin{array}{c}
x_{p}(k) \\
\dot{x}_{p}(k) \\
\ddot{x}_{p}(k)
\end{array}\right]=\left[\begin{array}{ccc}
1 & 0 & 0 \\
0 & 1 & 0 \\
0 & 0 & 1
\end{array}\right]\left[\begin{array}{c}
x_{p}(k-1) \\
\dot{x}_{p}(k-1) \\
\ddot{x}_{p}(k-1)
\end{array}\right]
$$




$$
\left[\begin{array}{c}
x_{o}(k) \\
\dot{x}_{o}(k)
\end{array}\right]=\left[\begin{array}{cc}
1 & 0 \\
0 & 1
\end{array}\right]\left[\begin{array}{c}
x_{o}(k) \\
\dot{x}_{o}(k)
\end{array}\right]
$$

where the position and the orientation $x_{p}(k), x_{o}(k)$ are measured by exoskelton, and the acceleration and the turn rate $\ddot{x}_{p}(k), \dot{x}_{o}(k)$ are measured by IMU. Although the velocity $\dot{x}_{p}(k)$ can be estimated by the Kalman filter in the state model, the integration of acceleration will cause a great cumulative error. Thus, the measurement value of the velocity $\dot{x}_{p}(k)$ is also provided in the measurement model using the measurement value of the position, and the measurement noise covariance of velocity is estimated using the measurement noise covariance of position.

\section{Kalman Filter}

Kalman filters are widely used in sensor fusion applications. By data fusion with an IMU using a Kalman filter, we are expecting to improve the performance of an arm exoskeleton.

Based on the state models and measurement models, the following linear time-invariant stochastic difference equations, which represent the estimation and the measurement, are used to implement the Kalman filter:

$$
x(k)=\Phi(T) x(k-1)+\omega(k)
$$

$$
y(k)=C x(k)+v(k)
$$

where $\omega(k)$ and $v(k)$ account for process and measurement noise, respectively. They are assumed to be drawn from a zero mean multivariate normal distribution with covariance $Q(k)$ and $R(k)$.

\section{EXPERIMENT AND RESULTS}

\section{A. Expriment Setup}

As shown in Fig. 3, the IMU is equipped at the end of the exoskeleton. The performance of the proposed method is evaluated by an experiment using an OTS with six cameras. The OTS which is much more accurate than the exoskeleton is used as a reference. The trajectory of exoskeleton's end detected by exoskeleton system and data fusion system are both compared with the trajectory detected by the optical tracking system.

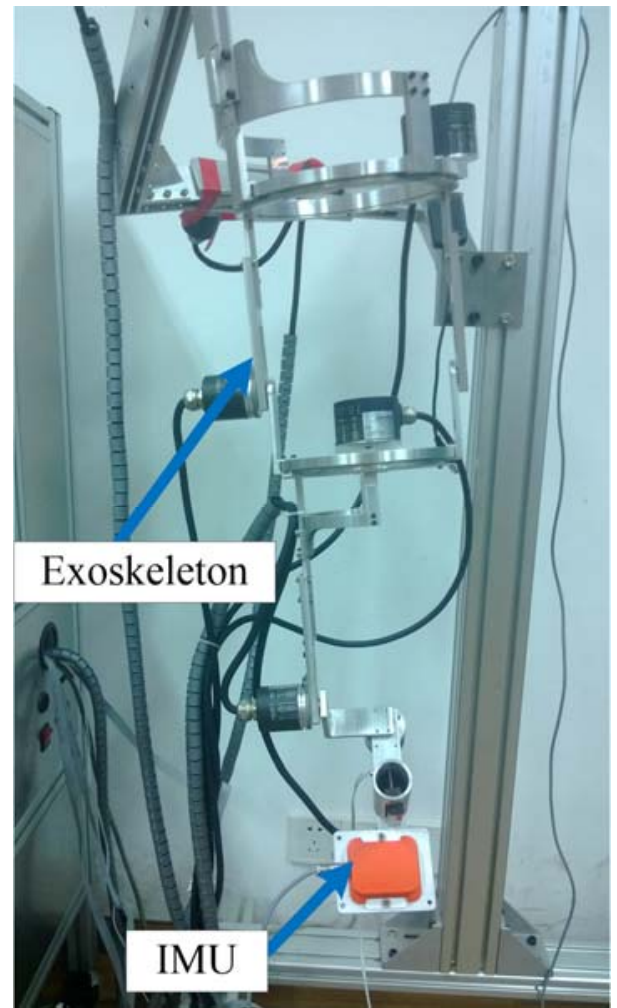

Figure 3. Experiment setup.

\section{B. Result}

Fig. 4 shows the acceleration noise of IMU after the compensation of gravity. Fig. 5 and Fig. 6 show the result of exoskeleton and data fusion compare with OTS, which indicate that the data fusion system has better performance than the exoskeleton system.

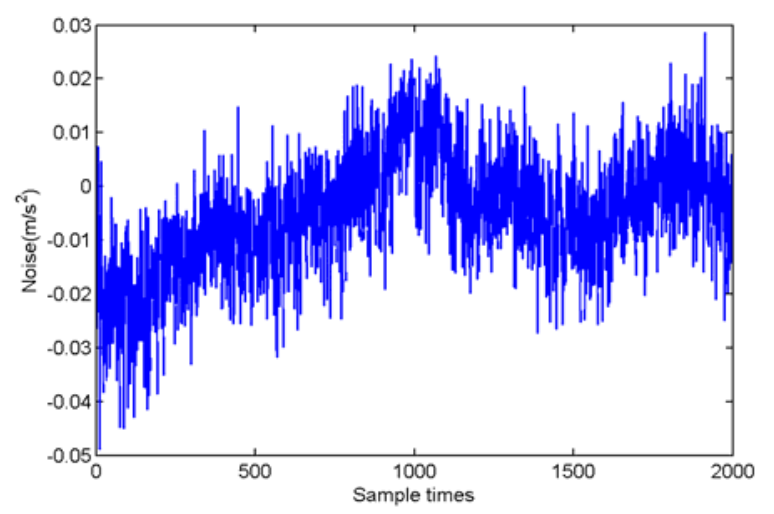

Figure 4. Acceleration noise of IMU. 


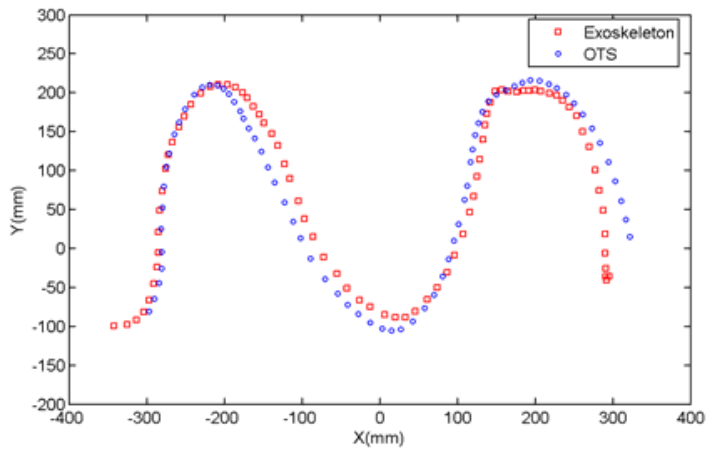

Figure 5. Result of exoskeleton.

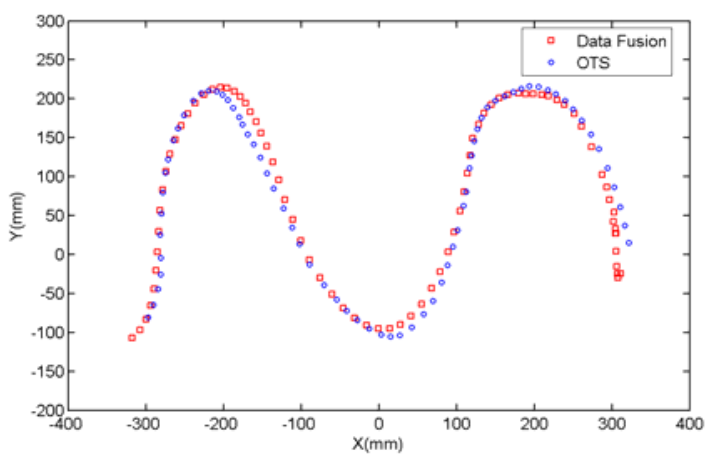

Figure 6. Result of data fusion.

\section{CONCLUSION AND DISCUSSION}

A method to improve the performance of an arm exoskeleton using data fusion with an inertial measurement unit was proposed. After the description of the system, the data fusion method was described in detail. The capability of the developed approach was proved by experimental results. In the proposed method, the noises of the sensor systems are treated as zero mean, Gaussian and white, thus Kalman filter is used for data fusion. However, considering the influence factors to the error of the exoskeleton, the exoskeleton system may not be treated as linear. For further studies, nonlinear filters, such as extended Kalman filter, are recommended for data fusion.

\section{REFERENCES}

[1] A. Gupta and M. K. O'Malley, "Design of a haptic arm exoskeleton for training and rehabilitation," Mechatronics, IEEE/ASME Transactions on, vol. 11, pp. 280-289, 2006.

[2] A. Gupta, M. K. O'Malley, V. Patoglu, and C. Burgar, "Design, control and performance of RiceWrist: A force feedback wrist exoskeleton for rehabilitation and training," The International Journal of Robotics Research, vol. 27, pp. 233-251, 2008.

[3] T. Nef and R. Riener, "ARMin-Design of a novel arm rehabilitation robot," in Rehabilitation Robotics, 2005. ICORR 2005. 9th International Conference on, 2005, pp. 57-60.

[4] Y. Ren, H. S. Park, and L. Q. Zhang, "Developing a whole-arm exoskeleton robot with hand opening and closing mechanism for upper limb stroke rehabilitation," in Rehabilitation Robotics, 2009. ICORR 2009. IEEE International Conference on, 2009, pp. 761-765.

[5] Z. F. Syed, P. Aggarwal, X. Niu et al., "Civilian vehicle navigation: required alignment of the inertial sensors for acceptable navigation accuracies,” Vehicular Technology, IEEE Transactions on, vol. 57, no. 6, pp. 3402-3412, 2008.

[6] Y. Dong, P. Zwahlen, A. M. Nguyen et al., "High performance inertial navigation grade sigma-delta MEMS accelerometer." pp. 3236.

[7] X. Yun, J. Calusdian, E. R. Bachmann et al., "Estimation of Human Foot Motion During Normal Walking Using Inertial and Magnetic Sensor Measurements," Instrumentation and Measurement, IEEE Transactions on, vol. 61, no. 7, pp. 2059-2072, 2012.

[8] H. Zhou, and H. Hu, "Reducing drifts in the inertial measurements of wrist and elbow positions," Instrumentation and Measurement, IEEE Transactions on, vol. 59, no. 3, pp. 575-585, 2010.

[9] P. Zhang, J. Gu, E. E. Milios et al., "Navigation with IMU/GPS/digital compass with unscented Kalman filter." pp. 14971502.

[10] S. Sukkarieh, E. M. Nebot, and H. F. Durrant-Whyte, "A high integrity IMU/GPS navigation loop for autonomous land vehicle applications," Robotics and Automation, IEEE Transactions on, vol. 15, no. 3, pp. 572-578, 1999.

[11] F. Caron, E. Duflos, D. Pomorski et al., "GPS/IMU data fusion using multisensor Kalman filtering: introduction of contextual aspects,” Information Fusion, vol. 7, no. 2, pp. 221-230, 2006.

[12] H. Ren, D. Rank, M. Merdes et al., "Multisensor Data Fusion in an Integrated Tracking System for Endoscopic Surgery," Information Technology in Biomedicine, IEEE Transactions on, vol. 16, no. 1, pp. 106-111, 2012.

[13] E. Foxlin, Y. Altshuler, L. Naimark et al., "FlightTracker: a novel optical/inertial tracker for cockpit enhanced vision." pp. 212-221.

[14] Y. Tao, and H. Hu, "A novel sensing and data fusion system for 3-D arm motion tracking in telerehabilitation," Instrumentation and Measurement, IEEE Transactions on, vol. 57, no. 5, pp. 1029-1040, 2008. 